\title{
Supplementary materials for "Hydrochemical
}

\section{and Mineralogical Evolution through Evaporitic}

\section{Processes in Atacama Waters"}

Authors list: Fermin Otálora ${ }^{1}$, Joaquín Criado-Reyes ${ }^{1}$, Magi Baselga $^{2}$, Angels Canals ${ }^{2}$, Cristobal Verdugo-Escamilla ${ }^{1}$, Juan Manuel García Ruiz ${ }^{* 1}$

Authors Affiliation:

${ }^{1}$ Laboratorio de Estudios Cristalográficos, IACT, CSIC/UGr, Av. Las Palmeras 4, Armilla, 18100, Granada, Spain.

${ }^{2}$ Departamento de Mineralogía, Petrología y Geología Aplicada, Facultat de Geologia, Universidad de Barcelona, C/Martí i Franques s/n.

Corresponding Author info: Juan Manuel García Ruiz, Consejo superior de Investigaciones Científicas, Laboratorio de Estudios Cristalográficos, IACT. Av. Las Palmeras 4. Armilla, Granada, ES 18100. Email: juanmanuel.garcia@csic.es

\section{CLIMATOLOGY AND HYDROLOGY}

Atacama Desert is considered the most arid area in Earth. It extends $1000 \mathrm{~km}$ from north to south and it is up to $150 \mathrm{~km}$ wide limiting with the Coastal Range on the west and with the Andean Range on the east: Atacama's desert conditions are due to convergence of the 
cold Humboldt Sea current from the south with dry air coming from the equator through high altitude Hadley cell system, forming a thermal inversion with a characteristic dense fog on the coast that doesn't spread over the Coastal Range ${ }^{1}$. The Coastal Range also increases the rain shadow desert effect preventing rainfall from the east. At present rainfall increases to the east with altitude, and there is hardly any rain under $2000 \mathrm{~m}$ above sea level. From December to February, vapor charged air masses reach the plateau from the Amazons basin to the east and convection triggered rainfall may occur. Only runoff seepage waters and water in dense fog form (camanchaca) reach the Central Depressions. Minor rainfall events, scattered within last decades, account for less than one millimeter of water per year on the Central Depression. Figure 2 shows the evolution of various climatologic indicators during the last 5 years at the Calate village, close to the area of study. Minimum temperature in the region ranges from around $12{ }^{\circ} \mathrm{C}$ in June to around $22{ }^{\circ} \mathrm{C}$ in January. The range for maximum temperatures is around $25-28{ }^{\circ} \mathrm{C}$ in the same months. Daily temperature changes are much larger in winter than in summer. Rain is very low and seasonal, with maxima typically during the first part of the year, typically less than $3 \mathrm{~mm}$ in January-March. Seasonal variations are also observed in humidity and in maximum wind speed, although not in the average wind speed. Table S1 summarizes the climatic data in the region during the two periods when data and samples were collected for our study.

Table S1: Climatologic values during the two field studies and sampling

\begin{tabular}{ccc} 
& $2011-10$ & $2012-03$ \\
\hline $\mathrm{T}_{\min }\left({ }^{\circ} \mathrm{C}\right)$ & 18 & 23 \\
\hline $\mathrm{T}_{\mathrm{avg}}\left({ }^{\circ} \mathrm{C}\right)$ & 22 & 25 \\
\hline $\mathrm{T}_{\max }\left({ }^{\circ} \mathrm{C}\right)$ & 25 & 27 \\
\hline
\end{tabular}




\begin{tabular}{ccc}
\hline rain days & 0 & 2 \\
\hline rain $(\mathrm{mm})$ & 0.1 & 1.6 \\
\hline Wind $_{\mathrm{avg}}(\mathrm{miles} / \mathrm{h})$ & 6.3 & 6.0 \\
\hline Wind $_{\max }(\mathrm{miles} / \mathrm{h})$ & 10.1 & 9.6 \\
\hline Atm. press $(\mathrm{mbar})$ & 1014.1 & 1011.5 \\
\hline cloud coverage $\%$ & 2 & 11 \\
\hline
\end{tabular}

There is no permanent surface water runoff reaching the Salar de Llamara area. Rainfall increases to the east with altitude from $1 \mathrm{~mm} / \mathrm{year}$ in the Salar to about $200 \mathrm{~mm} / \mathrm{year}$ on high Andean Range areas, so runoff and, mostly, seepage waters reach Salar de Llamara from the Andean Ranges on its easterly margin, where the salt crust onlaps the Prerange alluvial fans. Salar de Llamara aquifer works both as a confined aquifer on its deeper part and as an unconfined aquifer near the surface. The confinement layer varies in depth (up to 100 meters $)^{2}$. Total average inflow add up for Salar de Llamara basin is $309 \mathrm{~L} / \mathrm{s}$, while total evaporation is $209 \mathrm{~L} / \mathrm{s}^{3}$. Outflows of Salar de Llamara through Quebrada Amarga are, in average, $100 \mathrm{~L} / \mathrm{s}$ of water that springs out on its southern margin through the Quebrada Amarga Canyon.

The shallow phreatic zone waters dissolves part of the evaporites forming cavities that may collapse as a sink hole. This sink holes, locally known as Puquios, are small depressions on an evaporite substrate that may reach the phreatic zone and host ephemeral to perennial little lakes, colonized by halotolerant organisms and where copious dissolution and precipitation cycles may end up forming stromatolite-like structures. Puquios de Huatacondo are placed where Quebrada de Maní, Quebrada Sipuca and other minor drainage basins meets Llamara's salt crust. Water slowly migrate southwards, sweeping the Puquios de Huatacondo area (Fig. S1). The continuous evaporation reduces the size of Puquios, creating white salt crusts in the margin. Water column varies periodically during the year, as seen in figure 2. The bottom curve (labelled R3N2) correspond to seasonal 
variations of about $25 \mathrm{~cm}$ and corresponds to Puquio 2, the one we have investigated in depth and the subject of the following sections.

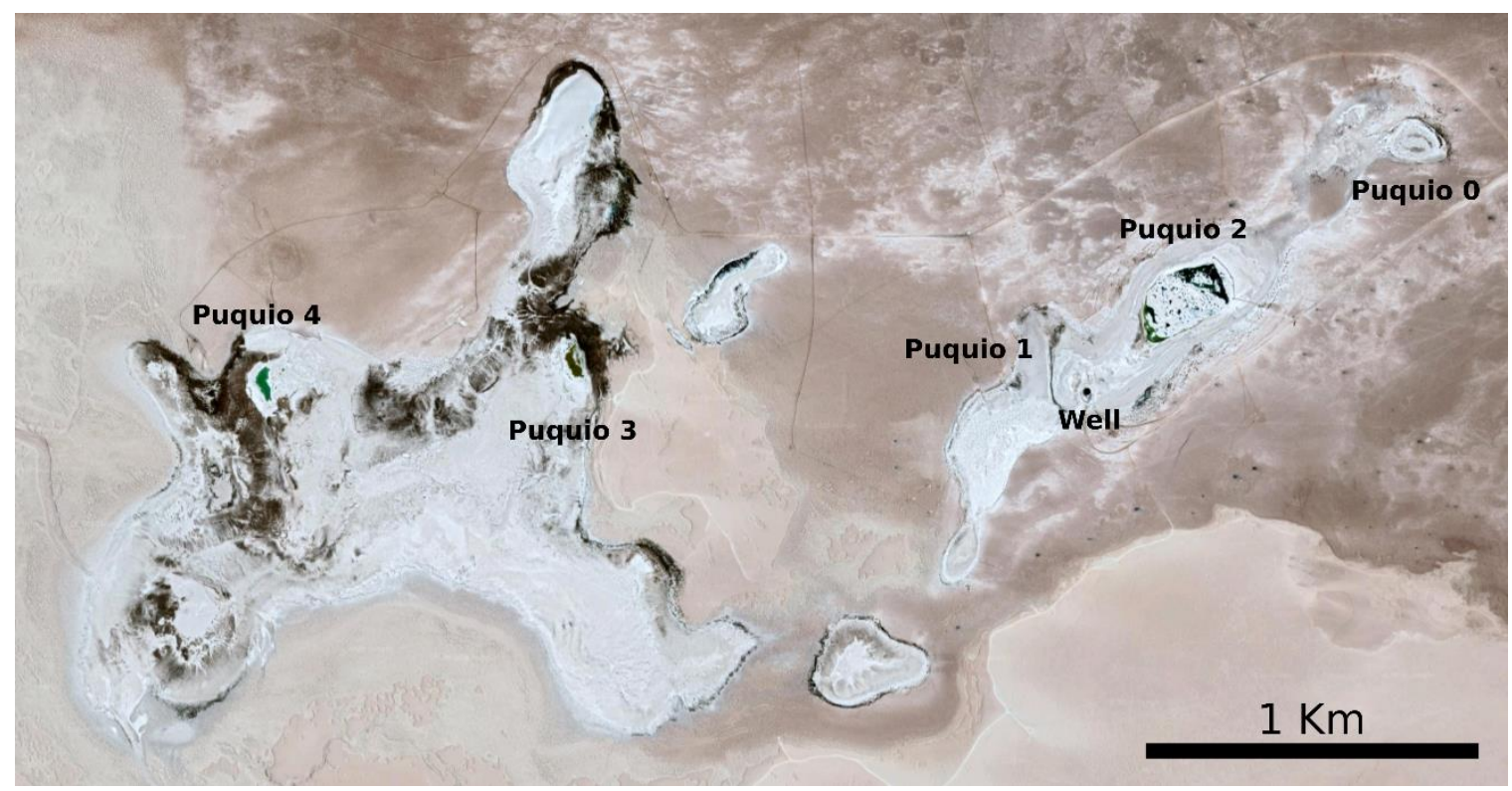

Figure S1: Aerial view of the Puquíos de Huatacondo. Coordinates of the center are $21^{\circ} 16^{\prime} 17.59^{\prime \prime} \mathrm{S}, 69^{\circ} 37^{\prime} 34.01^{\prime \prime} \mathrm{W}$. The puquíos studied during the field trips are labelled as well as the artificial well sampled (point $\mathrm{P}$ in figure 9).

\section{IN-SITU CHARACTERIZATION OF BRINES}

Table S2: Data collected in-situ from 53 sampling points (indicated in figure 4) in Puquío 2 during two field trips in October 2011 and March 2012. The "code" columns identifies the samples used in chemical analyses and corresponds to the equivalent column in tables S4-S8. Geolocalization data is provided in columns "latitude" and "longitude". The "depth" column contains the position of the measurement probe at the surface (top) or the bottom of the pond; points indicated as "unique" contain a single measurement representative of both surface and bottom, most times this is due to the small depth of the sampled pond. The remaining columns contain the measurements obtained using a Hanna 
HI 9828 multiparametric probe: " $\mathrm{T}$ " is the temperature in centigrade degrees $\left( \pm 0.015^{\circ} \mathrm{C}\right)$,

" $\mathrm{pH}$ " the $\mathrm{pH}( \pm 0.02)$, "ORP” is the oxidation/reduction potencial in $\mathrm{mV}( \pm 0.1 \mathrm{mV})$, "DO"

is the percent of dissolved oxygen $( \pm 3 \%)$, and "TDS" is the total dissolved solids in $\mathrm{mg} / \mathrm{L}$

\section{$( \pm 10 \mathrm{mg} / \mathrm{L})$}

\begin{tabular}{|c|c|c|c|c|c|c|c|c|c|c|}
\hline $\mathrm{N}$ & Code & latitude & longitude & date & depth & $\mathrm{T}$ & $\mathrm{pH}$ & ORP & DO & TDS \\
\hline \multirow[t]{4}{*}{0} & & $-21^{\circ} 16^{\prime} 12.17^{\prime \prime}$ & $-69^{\circ} 37^{\prime} 04.66^{\prime \prime}$ & oct-11 & bottom & 36.75 & 8.3 & -39.4 & 318 & 33060 \\
\hline & & & & oct-11 & top & 27.25 & 8.01 & -9.2 & 95 & 23030 \\
\hline & & & & mar-12 & bottom & 32.19 & 8.5 & -233.5 & 209 & 34380 \\
\hline & & & & mar-12 & top & 30.06 & 8.13 & -250.8 & 109 & 25190 \\
\hline 1 & & $-21^{\circ} 16^{\prime} 09.82^{\prime \prime}$ & $-69^{\circ} 37^{\prime} 04.29^{\prime \prime}$ & oct-11 & unique & 29.17 & 7.71 & -49.7 & 122 & 69590 \\
\hline \multirow[t]{4}{*}{2} & & $-21^{\circ} 16^{\prime} 09.85^{\prime \prime}$ & $-69^{\circ} 37^{\prime} 04.44^{\prime \prime}$ & oct-11 & bottom & 28.61 & 7.65 & 21.1 & 64 & 71070 \\
\hline & & & & oct-11 & top & 25.92 & 7.98 & 19.5 & 48 & 66800 \\
\hline & & & & mar-12 & bottom & 31.43 & 7.55 & -236.7 & 98 & 83270 \\
\hline & & & & mar-12 & top & 29.41 & 7.94 & -210.1 & 91 & 73980 \\
\hline \multirow[t]{2}{*}{3} & & $-21^{\circ} 16^{\prime} 11.38^{\prime \prime}$ & $-69^{\circ} 37^{\prime} 02.80^{\prime \prime}$ & oct-11 & bottom & 37.7 & 7.45 & -8.7 & 68 & 60390 \\
\hline & & & & oct-11 & top & 22.66 & 8.14 & 24.7 & 68 & 26230 \\
\hline \multirow[t]{3}{*}{4} & & $-21^{\circ} 16^{\prime} 12.39^{\prime \prime}$ & $-69^{\circ} 37^{\prime} 03.44^{\prime \prime}$ & oct-11 & bottom & 38.55 & 7.99 & 93.4 & 202 & 37520 \\
\hline & & & & mar-12 & bottom & 42.68 & 6.81 & -511.7 & 2 & 72760 \\
\hline & & & & mar-12 & top & 22.36 & 7.38 & -341.7 & 40 & 26130 \\
\hline 5 & & $-21^{\circ} 16^{\prime} 12.20^{\prime \prime}$ & $-69^{\circ} 37^{\prime} 02.76^{\prime \prime}$ & oct-11 & bottom & 45.39 & 7.21 & 137.8 & 160 & 64320 \\
\hline \multirow[t]{4}{*}{6} & & $-21^{\circ} 16^{\prime} 10.39^{\prime \prime}$ & $-69^{\circ} 37^{\prime} 04.44^{\prime \prime}$ & oct-11 & bottom & 36.53 & 7.64 & -136 & 127 & 65390 \\
\hline & & & & oct-11 & top & 25.91 & 8 & -65.4 & 60 & 51430 \\
\hline & & & & mar-12 & bottom & 43.48 & 7.6 & -374.2 & 31 & 82950 \\
\hline & & & & mar-12 & top & 28.93 & 8.07 & -284 & 96 & 52790 \\
\hline \multirow[t]{4}{*}{7} & & $-21^{\circ} 16^{\prime} 11.38^{\prime \prime}$ & $-69^{\circ} 37^{\prime} 05.42^{\prime \prime}$ & oct-11 & bottom & 32.07 & 8.39 & -8.7 & 104 & 26220 \\
\hline & & & & oct-11 & top & 26.37 & 8.12 & 6.5 & 61 & 23170 \\
\hline & & & & $\operatorname{mar}-12$ & bottom & 29.48 & 8.07 & -230.8 & 74 & 28220 \\
\hline & & & & mar-12 & top & 29.02 & 8.24 & -283 & 75 & 28430 \\
\hline \multirow[t]{4}{*}{8} & & $-21^{\circ} 16^{\prime} 12.08^{\prime \prime}$ & $-69^{\circ} 37^{\prime} 04.00^{\prime \prime}$ & oct-11 & bottom & 27.28 & 8.3 & 95.8 & 149 & 43310 \\
\hline & & & & oct-11 & top & 24.54 & 8.34 & 72 & 52 & 23170 \\
\hline & & & & mar-12 & bottom & 27.83 & 8.08 & -288.1 & 34 & 58790 \\
\hline & & & & mar-12 & top & 27.03 & 8.46 & -273.3 & 93 & 30510 \\
\hline \multirow[t]{2}{*}{9} & & $-21^{\circ} 16^{\prime} 09.85^{\prime \prime}$ & $-69^{\circ} 37^{\prime} 03.49^{\prime \prime}$ & oct-11 & bottom & 27.08 & 7.75 & 14.8 & 69 & 73710 \\
\hline & P11-8 & & & oct-11 & top & 25.36 & 7.82 & 15.6 & 73 & 72610 \\
\hline \multirow[t]{2}{*}{$\mathrm{P}$} & $12-8 b$ & & & mar-12 & bottom & 36.46 & 7.61 & -164.9 & 99 & 84090 \\
\hline & $\mathrm{P} 12-8$ & & & mar-12 & top & 28.05 & 7.9 & -165.4 & 91 & 77700 \\
\hline \multirow[t]{4}{*}{10} & & $-21^{\circ} 16^{\prime} 10.25^{\prime \prime}$ & $-69^{\circ} 37^{\prime} 03.98^{\prime \prime}$ & oct-11 & bottom & 35.34 & 7.62 & 31.1 & 89 & 66320 \\
\hline & & & & oct-11 & top & 27.13 & 8.05 & 30.9 & 52 & 56490 \\
\hline & & & & mar-12 & bottom & 39.73 & 7.45 & -293.9 & 39 & 80950 \\
\hline & & & & mar-12 & top & 28.19 & 8.07 & -249.9 & 95 & 64440 \\
\hline 11 & & $12.34^{\prime \prime}$ & '05.04“ & oct-11 & bottom & 26.14 & 8.29 & -12.4 & 86 & 20460 \\
\hline \multirow[t]{3}{*}{12} & & $-21^{\circ} 16^{\prime} 11.73^{\prime \prime}$ & $-69^{\circ} 37^{\prime} 04.71^{\prime \prime}$ & oct-11 & bottom & 42.17 & 7.84 & 64.1 & 155 & 51690 \\
\hline & & & & mar-12 & bottom & 38.71 & 7.85 & -178 & 181 & 65310 \\
\hline & & & & mar-12 & top & 27.35 & 8.24 & -181.5 & 110 & 32910 \\
\hline 13 & & $-21^{\circ} 16^{\prime} 12.28^{\prime \prime}$ & $-69^{\circ} 37^{\prime} 04.36^{\prime \prime}$ & oct-11 & bottom & 35.83 & 8.11 & 30 & 166 & 39240 \\
\hline \multirow[t]{4}{*}{14} & & $-21^{\circ} 16^{\prime} 10.72^{\prime \prime}$ & $-69^{\circ} 37^{\prime} 03.62^{\prime \prime}$ & oct-11 & bottom & 34.64 & 7.7 & 59 & 157 & 67380 \\
\hline & & & & oct-11 & top & 23.9 & 8.08 & 61.5 & 76 & 47120 \\
\hline & & & & mar-12 & bottom & 41.55 & 7.45 & -208.9 & 149 & 83450 \\
\hline & & & & mar-12 & top & 26.62 & 8.09 & -197 & 108 & 50810 \\
\hline 15 & & $-21^{\circ} 16^{\prime} 09.84^{\prime \prime}$ & $-69^{\circ} 37^{\prime} 03.20^{\prime \prime}$ & oct-11 & unique & 25.52 & 7.79 & 35.8 & 64 & 73520 \\
\hline \multirow[t]{4}{*}{16} & P11-4 & $-21^{\circ} 16^{\prime} 11.18^{\prime \prime}$ & $-69^{\circ} 37^{\prime} 03.71^{\prime \prime}$ & oct-11 & bottom & 47.42 & 7.25 & -184.9 & 37 & 74250 \\
\hline & P11-5 & & & oct-11 & top & 24.02 & 8.18 & -15 & 50 & 32980 \\
\hline & $\mathrm{P} 12-4$ & & & mar-12 & bottom & 38.26 & 7.46 & -194.4 & 195 & 79990 \\
\hline & P12-5 & & & mar-12 & top & 25.57 & 8.2 & -191.2 & 105 & 38090 \\
\hline 11 & & $-21^{\circ} 16^{\prime} 11.21^{\prime \prime}$ & $-69^{\circ} 37^{\prime} 03.02^{\prime \prime}$ & oct-11 & unique & 29.24 & 8.32 & 14.2 & 140 & 28530 \\
\hline
\end{tabular}




\begin{tabular}{|c|c|c|c|c|c|c|c|c|c|c|}
\hline 18 & & $-21^{\circ} 16^{\prime} 10.27^{\prime \prime}$ & $-69^{\circ} 37^{\prime} 03.54^{\prime \prime}$ & oct-11 & unique & 25.48 & 8.09 & 11.2 & 53 & 53190 \\
\hline 19 & & $-21^{\circ} 16^{\prime} 11.80^{\prime \prime}$ & $-69^{\circ} 37^{\prime} 03.11^{\prime \prime}$ & oct-11 & top & 26.28 & 8.31 & -20.9 & 71 & 20580 \\
\hline \multirow[t]{4}{*}{20} & & $-21^{\circ} 16^{\prime} 10.65^{\prime \prime}$ & $-69^{\circ} 37^{\prime} 04.37^{\prime \prime}$ & oct-11 & bottom & 34.46 & 7.53 & 17 & 108 & 69380 \\
\hline & & & & oct-11 & top & 24.66 & 8.14 & 22.3 & 56 & 42760 \\
\hline & & & & mar-12 & bottom & 37.56 & 7.7 & -224.2 & 41 & 76770 \\
\hline & & & & mar-12 & top & 27.58 & 8.14 & $\begin{array}{l}-196.8 \\
\end{array}$ & 89 & 46400 \\
\hline $21 \mathrm{P}$ & 11-oct & $-21^{\circ} 16^{\prime} 09.44^{\prime \prime}$ & $-69^{\circ} 37^{\prime} 02.92^{\prime \prime}$ & oct-11 & unique & 23.61 & 8.12 & 172.3 & 56 & 67450 \\
\hline \multirow[t]{2}{*}{22} & P11-9 & $-21^{\circ} 16^{\prime} 10.03^{\prime \prime}$ & $-69^{\circ} 37^{\prime} 02.89^{\prime \prime}$ & oct-11 & bottom & 24.67 & 7.86 & 45.2 & 41 & 72490 \\
\hline & & & & oct-11 & top & 24.63 & 7.85 & 46.7 & 75 & 72660 \\
\hline \multirow[t]{2}{*}{$\mathrm{P}$} & $12-9 \mathrm{~b}$ & & & mar-12 & bottom & 35.84 & 7.55 & -153.4 & 86 & 86380 \\
\hline & P12-9 & & & mar-12 & top & 28.16 & 7.93 & -158.1 & 90 & 77760 \\
\hline \multirow[t]{3}{*}{23} & & $-21^{\circ} 16^{\prime} 11.17^{\prime \prime}$ & $-69^{\circ} 37^{\prime} 04.22^{\prime \prime}$ & oct-11 & bottom & 44.64 & 7.37 & -135 & 86 & 56660 \\
\hline & & & & mar-12 & bottom & 38.13 & 7.65 & -206.2 & 57 & 62560 \\
\hline & & & & mar-12 & top & 26.94 & 8.16 & -191 & 87 & 39620 \\
\hline \multirow[t]{2}{*}{24} & & $-21^{\circ} 16^{\prime} 11.69^{\prime \prime}$ & $-69^{\circ} 37^{\prime} 02.92^{\prime \prime}$ & oct-11 & bottom & 49.22 & 7.32 & -264.4 & 15 & 72900 \\
\hline & & & & oct-11 & top & 24.44 & 8.08 & -108.8 & 77 & 26880 \\
\hline \multirow[t]{4}{*}{25} & $\mathrm{P} 11-2$ & $-21^{\circ} 16^{\prime} 12.29^{\prime \prime}$ & $-69^{\circ} 37^{\prime} 02.17^{\prime \prime}$ & oct-11 & bottom & 47.87 & 7.61 & 95.6 & 216 & 51360 \\
\hline & P11-3 & & & oct-11 & top & 23.77 & 8.22 & 22.2 & 122 & 25700 \\
\hline & $\mathrm{P} 12-2$ & & & mar-12 & bottom & 43.12 & 6.96 & -228.5 & 170 & 60970 \\
\hline & P12-3 & & & mar-12 & top & 26.4 & 7.89 & -203.1 & 120 & 32720 \\
\hline \multirow[t]{2}{*}{26} & & $-21^{\circ} 16^{\prime} 09.13^{\prime \prime}$ & $-69^{\circ} 37^{\prime} 03.22^{\prime \prime}$ & oct-11 & unique & 23.98 & 8.13 & 166.3 & 41 & 67490 \\
\hline & & & & mar-12 & unique & 30.83 & 7.97 & -183.7 & 39 & 89180 \\
\hline 27 & & $-21^{\circ} 16^{\prime} 11.41^{\prime \prime}$ & $-69^{\circ} 37^{\prime} 05.16^{\prime \prime}$ & oct-11 & top & 25.49 & 8.16 & -50.6 & 57 & 33440 \\
\hline \multirow[t]{2}{*}{28} & & $-21^{\circ} 16^{\prime} 11.76^{\prime \prime}$ & $-69^{\circ} 37^{\prime} 03.26^{\prime \prime}$ & mar-12 & bottom & 43.12 & 7.53 & -163.3 & 158 & 78730 \\
\hline & & & & mar-12 & top & 26.41 & 8.18 & -159.6 & 95 & 30480 \\
\hline \multirow[t]{2}{*}{29} & & $-21^{\circ} 16^{\prime} 13.33^{\prime \prime}$ & $-69^{\circ} 37^{\prime} 04.54^{\prime \prime}$ & oct-11 & unique & 24.87 & 8.41 & 27.3 & 65 & 19230 \\
\hline & & & & mar-12 & unique & 25.48 & 8.58 & -243.4 & 95 & 20550 \\
\hline \multirow[t]{2}{*}{30} & & $-21^{\circ} 16^{\prime} 12.86^{\prime \prime}$ & $03.54 “$ & oct-11 & unique & 25.54 & 8.21 & 58.8 & 60 & 19890 \\
\hline & & & & mar-12 & unique & 27.79 & 8.38 & -346.2 & 98 & 26730 \\
\hline \multirow[t]{2}{*}{31} & & $-21^{\circ} 16^{\prime} 11.76^{\prime \prime}$ & $-69^{\circ} 37^{\prime} 03.26^{\prime \prime}$ & mar-12 & bottom & 43.12 & 7.53 & -163.3 & 158 & 78730 \\
\hline & & & & mar-12 & top & 26.41 & 8.18 & -159.6 & 95 & 30480 \\
\hline 32 & & $-21^{\circ} 16^{\prime} 11.93^{\prime \prime}$ & $-69^{\circ} 37^{\prime} 05.60^{\prime \prime}$ & oct-11 & unique & 25.73 & 8.17 & 51 & 50 & 24010 \\
\hline \multirow[t]{3}{*}{33} & & $-21^{\circ} 16^{\prime} 12.64^{\prime \prime}$ & $-69^{\circ} 37^{\prime} 05.88^{\prime \prime}$ & oct-11 & top & 29.38 & 7.82 & 12.4 & 101 & 60220 \\
\hline & & & & mar-12 & bottom & 29.61 & 8.31 & -257.5 & 123 & 25340 \\
\hline & & & & mar-12 & top & 29.66 & 8.33 & -284.3 & 106 & 25420 \\
\hline 34 & & $-21^{\circ} 16^{\prime} 09.55^{\prime \prime}$ & $-69^{\circ} 37^{\prime} 03.29^{\prime \prime}$ & oct-11 & unique & 23.7 & 7.78 & 66.6 & 48 & 76430 \\
\hline 35 & & $-21^{\circ} 16^{\prime} 10.21^{\prime \prime}$ & $-69^{\circ} 37^{\prime} 02.40^{\prime \prime}$ & oct-11 & unique & 23.62 & 8.17 & 157.3 & 37 & 67620 \\
\hline 36 & P11-6 & $-21^{\circ} 16^{\prime} 09.97^{\prime \prime}$ & $-69^{\circ} 37^{\prime} 04.76^{\prime \prime}$ & oct-11 & bottom & 41.1 & 7.22 & -193.9 & 59 & 74760 \\
\hline & P11-7 & & & oct-11 & top & 24.65 & 7.55 & -88.2 & 39 & 53550 \\
\hline & P12-6 & & & mar-12 & bottom & 39.84 & 7.59 & -158.2 & 218 & 83230 \\
\hline & P12-7 & & & mar-12 & top & 26.72 & 8.13 & -170 & 100 & 50920 \\
\hline 37 & & $-21^{\circ} 16^{\prime} 12.28^{\prime \prime}$ & $-69^{\circ} 37^{\prime} 02.43^{\prime \prime}$ & oct-11 & bottom & 45.08 & 7.83 & 87.3 & 193 & 49530 \\
\hline 38 & & $-21^{\circ} 16^{\prime} 09.78^{\prime \prime}$ & $-69^{\circ} 37^{\prime} 03.76^{\prime \prime}$ & oct-11 & bottom & 25.67 & 7.95 & -7.5 & 44 & 70140 \\
\hline & & & & oct-11 & top & 25.73 & 7.96 & 16.2 & 48 & 70550 \\
\hline & & & & mar-12 & bottom & 27.65 & 7.79 & -178 & 51 & 91860 \\
\hline & & & & mar-12 & top & 29.44 & 7.87 & -177.8 & 77 & 80950 \\
\hline 39 & & $0.66^{\prime \prime}$ & $01.25 “$ & oct-11 & unique & 25.85 & 8.12 & -0.2 & 32 & 68330 \\
\hline 40 & & $-21^{\circ} 16^{\prime} 12.36^{\prime \prime}$ & $-69^{\circ} 37^{\prime} 06.19^{\prime \prime}$ & oct-11 & unique & 25.85 & 8.19 & 22.4 & 56 & 22490 \\
\hline 41 & & $-21^{\circ} 16^{\prime} 11.23^{\prime \prime}$ & $-69^{\circ} 37^{\prime} 05.12^{\prime \prime}$ & oct-11 & unique & 29.43 & 8.22 & -22.9 & 75 & 32080 \\
\hline $42 \mathrm{P}$ & 12-oct & $-21^{\circ} 16^{\prime} 11.28^{\prime \prime}$ & $-69^{\circ} 37^{\prime} 01.54^{\prime \prime}$ & mar-12 & unique & 30.89 & 7.97 & -175.7 & 63 & 89030 \\
\hline 43 & & $-21^{\circ} 16^{\prime} 10.70^{\prime \prime}$ & $-69^{\circ} 37^{\prime} 03.40^{\prime \prime}$ & oct-11 & bottom & 33.67 & 7.58 & 34.1 & 93 & 65720 \\
\hline & & & & oct-11 & top & 24.02 & 8.09 & 36.4 & 84 & 48700 \\
\hline & & & & mar-12 & bottom & 37.64 & 7.43 & -243.9 & 103 & 87440 \\
\hline & & & & mar-12 & top & 26.38 & 8.11 & -216.1 & 95 & 53970 \\
\hline 44 & P11-1 & $-21^{\circ} 16^{\prime} 13.26^{\prime \prime}$ & $-69^{\circ} 37^{\prime} 06.97^{\prime \prime}$ & oct-11 & unique & 25.02 & 8.47 & -27.1 & 46 & 18770 \\
\hline & P12-1 & & & mar-12 & unique & 29.1 & 8.3 & -230.4 & 105 & 19140 \\
\hline 45 & & $-21^{\circ} 16^{\prime} 11.43^{\prime \prime}$ & $-69^{\circ} 37^{\prime} 03.50^{\prime \prime}$ & oct-11 & bottom & 40.38 & 7.39 & -214.7 & 126 & 57360 \\
\hline & & & & oct-11 & top & 24.53 & 8.07 & -101.4 & 93 & 26950 \\
\hline & & & & mar-12 & bottom & 35.98 & 7.76 & -274.2 & 43 & 69700 \\
\hline & & & & mar-12 & top & 25.46 & 8.18 & -215.9 & 83 & 30360 \\
\hline 46 & & $-21^{\circ} 16^{\prime} 12.90^{\prime \prime}$ & $-69^{\circ} 37^{\prime} 06.57^{“ \star}$ & oct-11 & unique & 24.85 & 8.27 & 19 & 43 & 20820 \\
\hline 47 & & $-21^{\circ} 16^{\prime} 08.80^{\prime \prime}$ & $-69^{\circ} 37^{\prime} 02.42^{\prime \prime}$ & oct-11 & unique & 30.02 & 7.95 & -0.7 & 57 & 68200 \\
\hline 48 & & $-21^{\circ} 16^{\prime} 11.26^{\prime \prime}$ & $-69^{\circ} 37^{\prime} 01.82^{\prime \prime}$ & oct-11 & unique & 26.41 & 8.1 & 168.3 & 56 & 67690 \\
\hline
\end{tabular}




\begin{tabular}{llllllllll}
49 & $-21^{\circ} 16^{\prime} 10.12^{\prime \prime}$ & $-69^{\circ} 37^{\prime} 03.81^{\prime \prime}$ & oct-11 & bottom & 38.85 & 7.64 & 14.2 & 186 & 71570 \\
\hline & & oct-11 & top & 26.58 & 8.04 & 17.9 & 72 & 58030 \\
\hline & & mar-12 & bottom & 41.07 & 7.57 & -184.2 & 204 & 84130 \\
\hline & & mar-12 & top & 29.16 & 8.02 & -197.4 & 97 & 67690 \\
\hline 50 & $-21^{\circ} 16^{\prime} 10.35^{\prime \prime}$ & $-69^{\circ} 37^{\prime} 01.74^{\prime \prime}$ & mar-12 & unique & 29.57 & 7.98 & -179.6 & 68 & 88540 \\
\hline 51 & $-21^{\circ} 16^{\prime} 09.26^{\prime \prime}$ & $-69^{\circ} 37^{\prime} 00.82^{\prime \prime}$ & oct-11 & unique & 24.91 & 8.12 & 12.1 & 34 & 68520 \\
\hline & & & mar-12 & unique & 29.84 & 7.97 & -172.4 & 58 & 88100 \\
\hline 52 & $-21^{\circ} 16^{\prime} 09.55^{\prime \prime}$ & $-69^{\circ} 37^{\prime} 04.33^{\prime \prime}$ & oct-11 & unique & 33.53 & 7.63 & -192.8 & 38 & 63690 \\
\hline
\end{tabular}



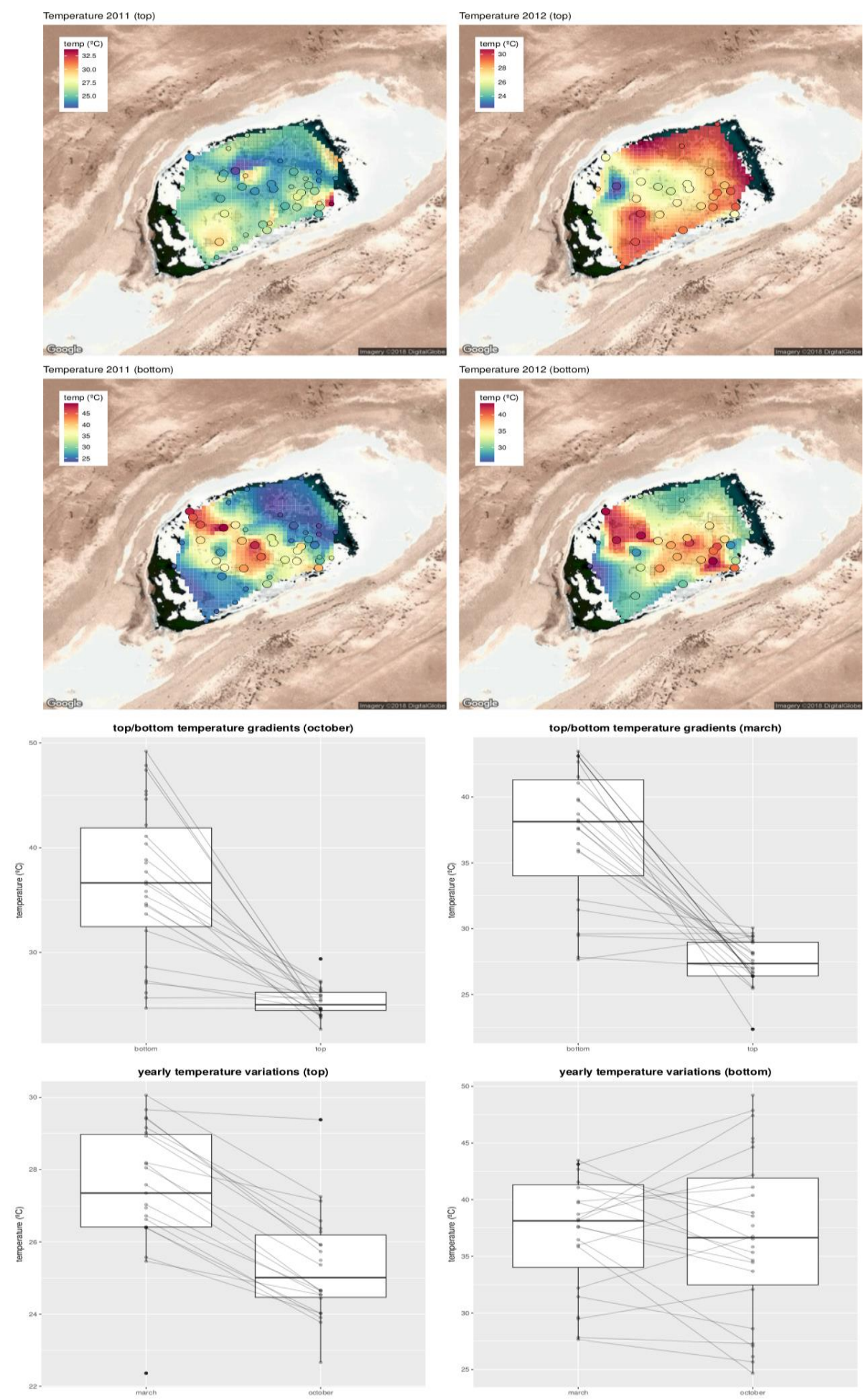

Figure S2: Spatial, seasonal and depth gradients of the in-situ measured temperature values. 

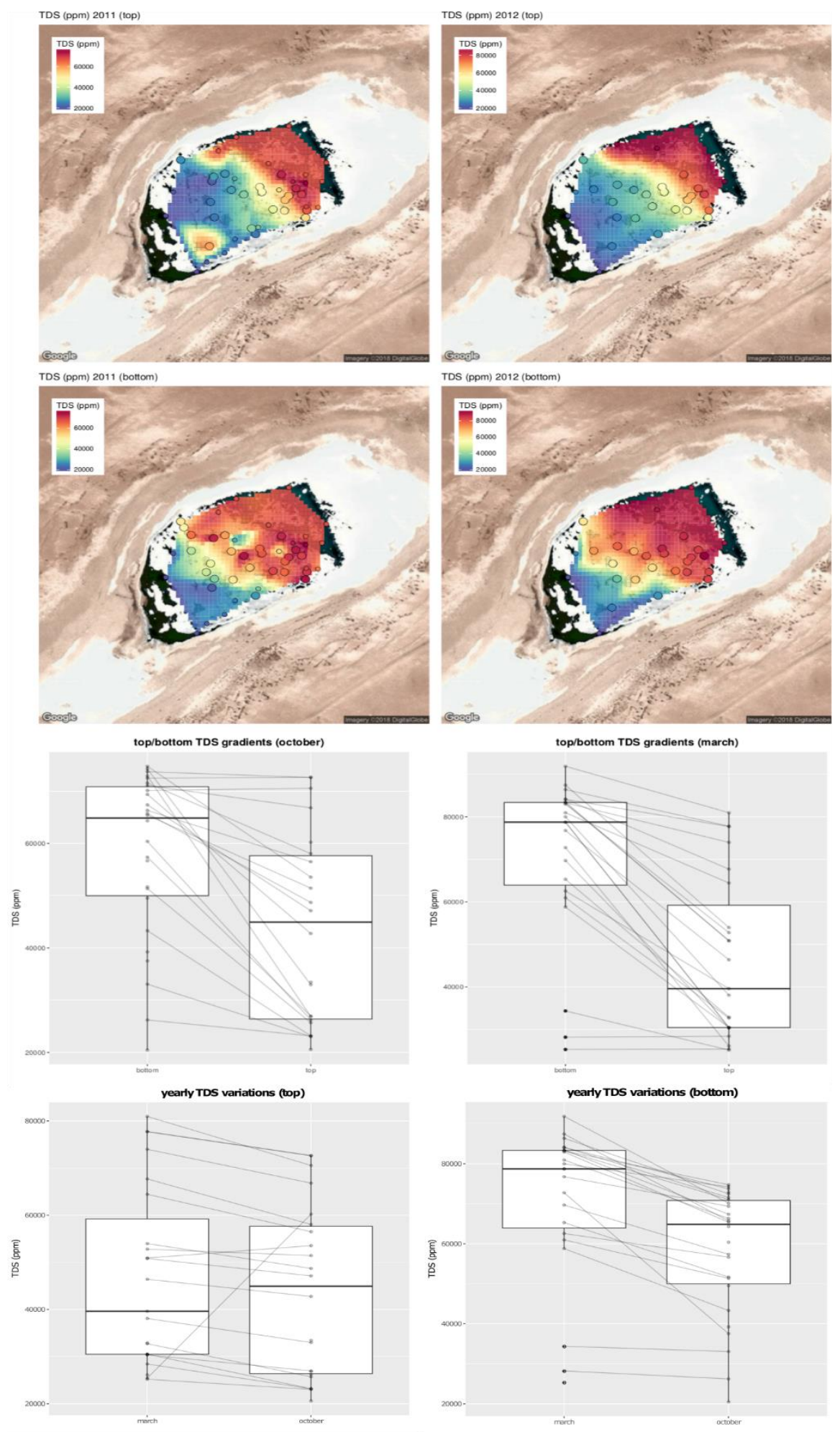

Figure S3: Spatial, seasonal and depth gradients of the in-situ measured total dissolved solids values. 


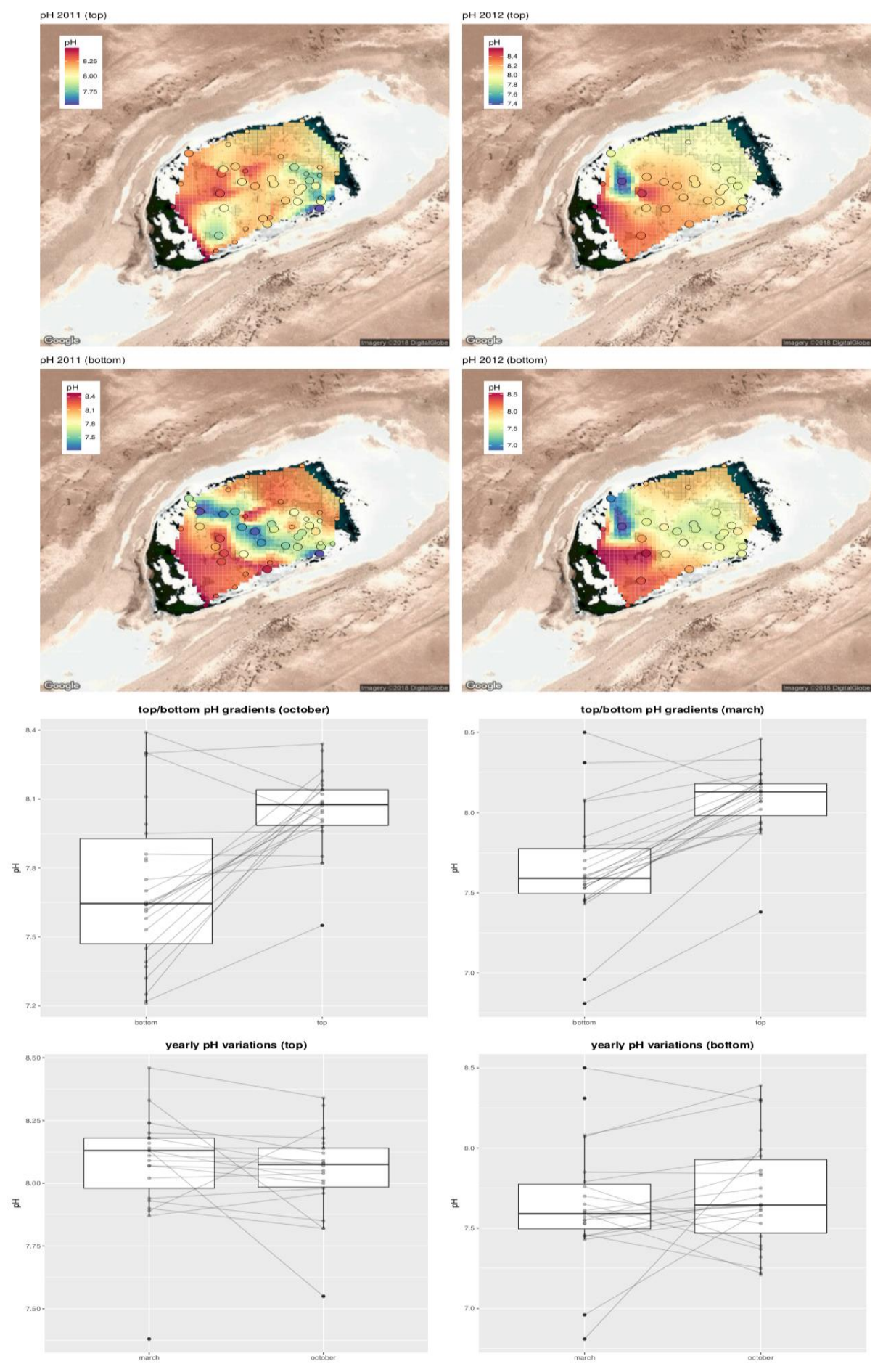

Figure S4: Spatial, seasonal and depth gradients of the in-situ measured $\mathrm{pH}$ values. 

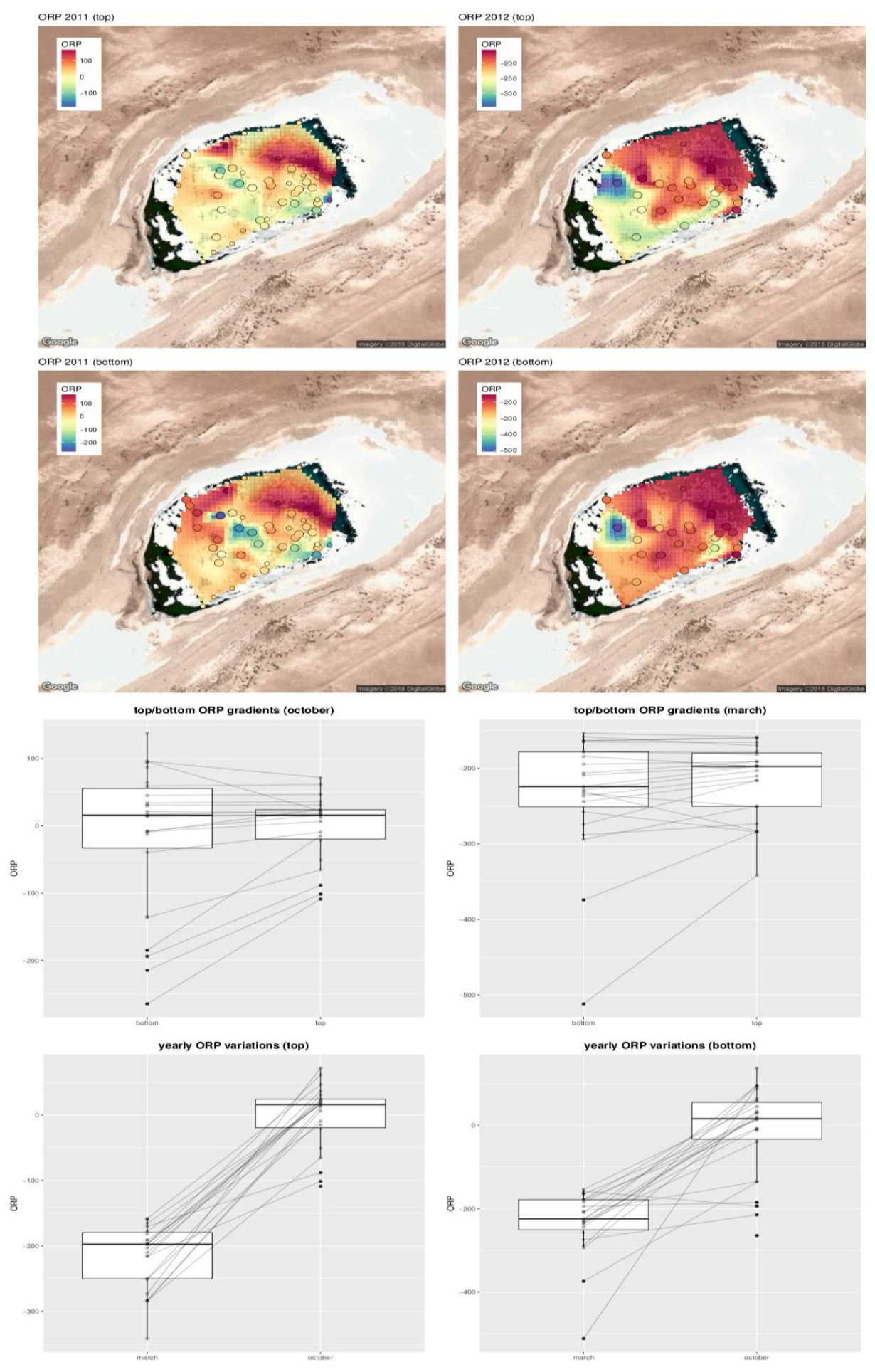

Figure S5: Spatial, seasonal and depth gradients of the in-situ measured oxidationreduction potential values. 


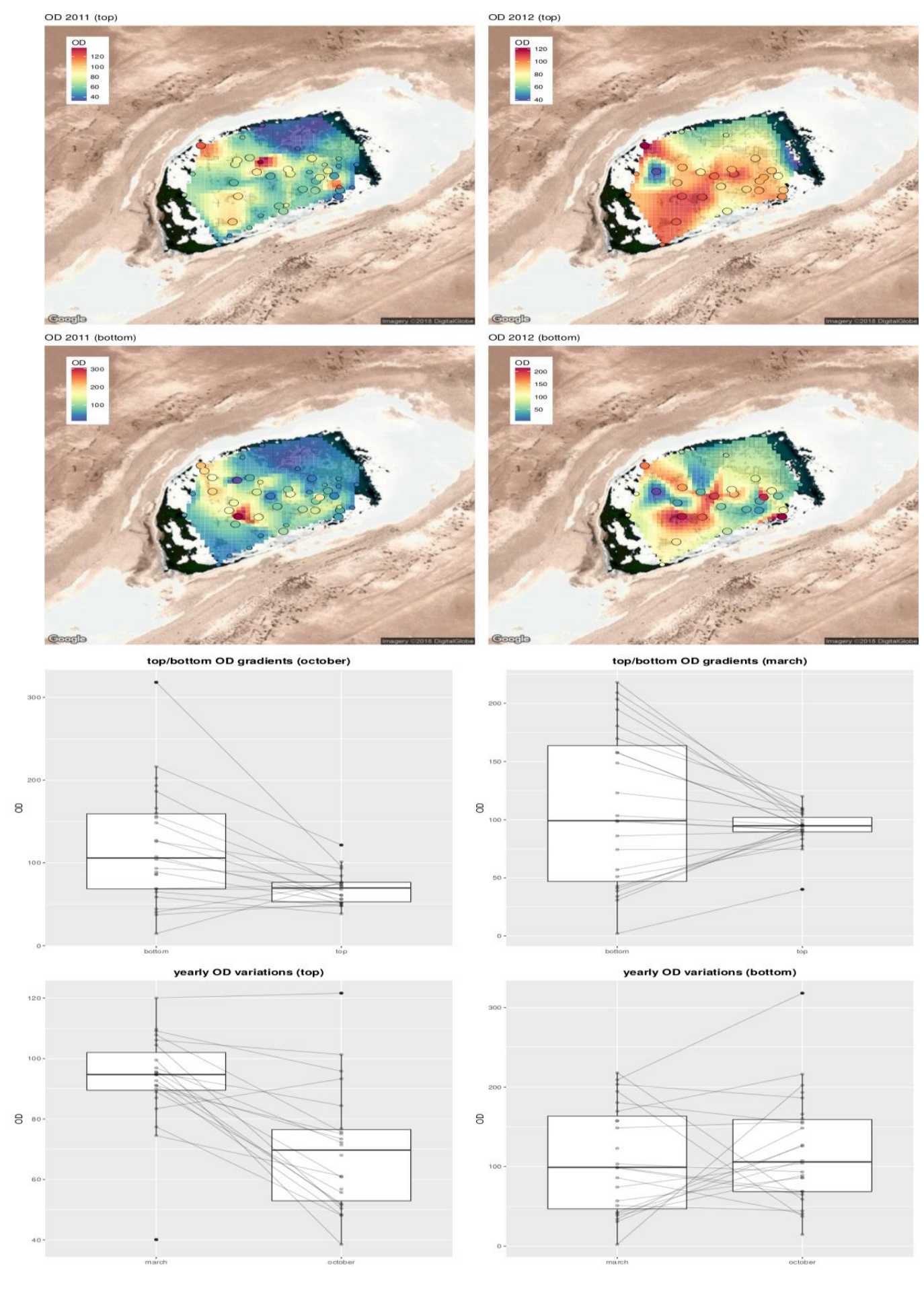

Figure S6: Spatial, seasonal and depth gradients of the in-situ measured oxygen dissolved values. 
Table S3: Tukey's statistics (lower-hinge, median, upper-hinge) for the data plotted in figures S3-S7. The two hinges are versions of the first and third quartile. ${ }^{4}$ "Exploratory Data Analysis". Section 2C.

\begin{tabular}{llllll} 
Quantity & date & position & lhinge & median & uhinge \\
\hline Temp & Oct-2011 & bottom & 32.07 & 36.64 & 42.17 \\
\hline & Oct-2011 & top & 24.44 & 25.01 & 26.28 \\
\hline & Mar-2012 & bottom & 34.02 & 38.13 & 41.31 \\
\hline $\mathrm{pH}$ & Mar-2012 & top & 26.41 & 27.35 & 28.98 \\
\hline & Oct-2011 & bottom & 7.45 & 7.65 & 7.95 \\
\hline & Oct-2011 & top & 7.98 & 8.08 & 8.14 \\
\hline Mar-2012 & bottom & 7.50 & 7.59 & 7.78 \\
\hline ORP & Mar-2012 & top & 7.98 & 8.13 & 8.18 \\
\hline & Oct-2011 & bottom & -39.4 & 15.9 & 59.0 \\
\hline Oct-2011 & top & -20.9 & 15.9 & 24.7 \\
\hline Mar-2012 & bottom & -250.7 & -224.2 & -178.0 \\
\hline MD & Oct-2012 & top & 250.4 & -197.4 & -179.7 \\
\hline & bct-2011 & top & 52.0 & 69.7 & 76.8 \\
\hline Mar-2012 & bottom & 46.9 & 99.1 & 163.6 \\
\hline TDS & Mar-2012 & top & 89.5 & 94.7 & 102.0 \\
\hline & Oct-2011 & bottom & 49530 & 64855 & 71070 \\
\hline & Oct-2011 & top & 26230 & 44940 & 58030 \\
\hline Mar-2012 & bottom & 63935 & 78730 & 83360 \\
\hline Mar-2012 & top & 30480 & 39620 & 59205 \\
\hline & & & & &
\end{tabular}

\section{MINERALOGY OF SAMPLES}

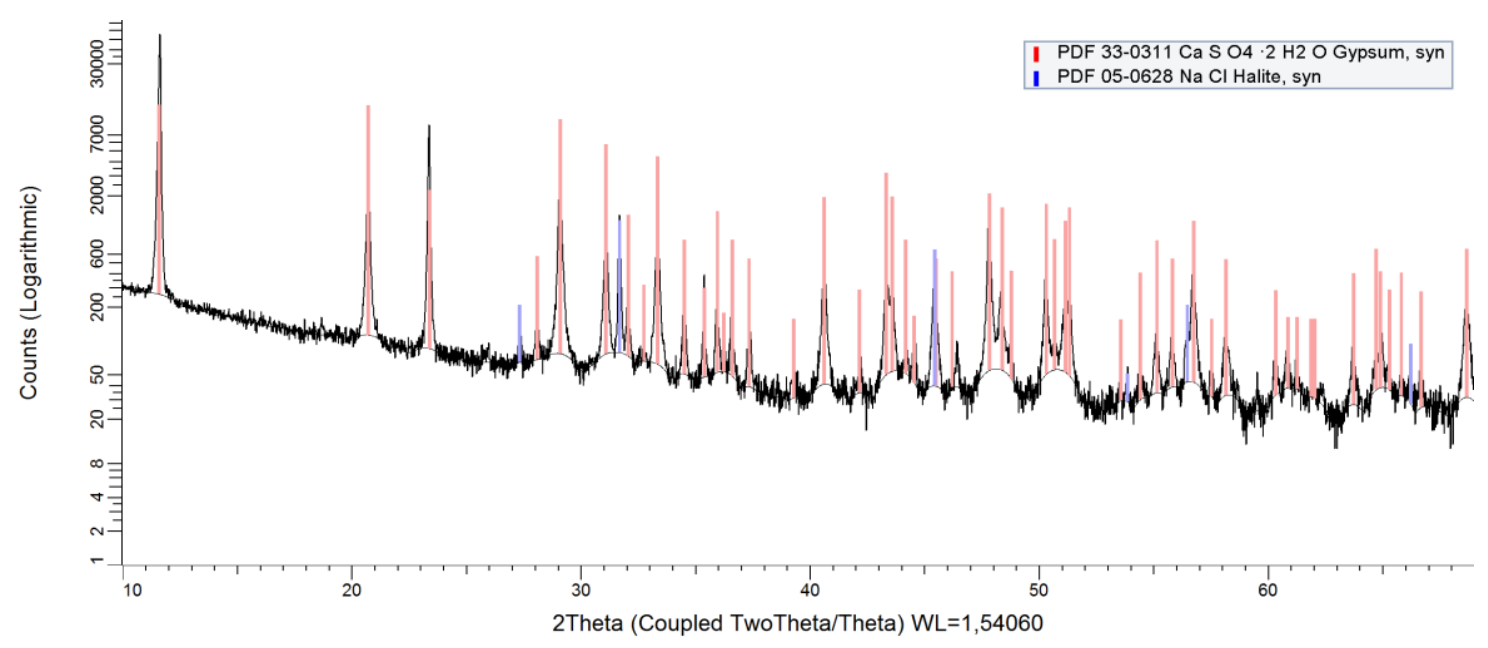

Figure S7: Representative diffractogram of the millimetric to centimetric crystalline gypsum crystals grouped in radial aggregates at the bottom of the ponds. The sample is composed of almost pure gypsum with some minor halite. 


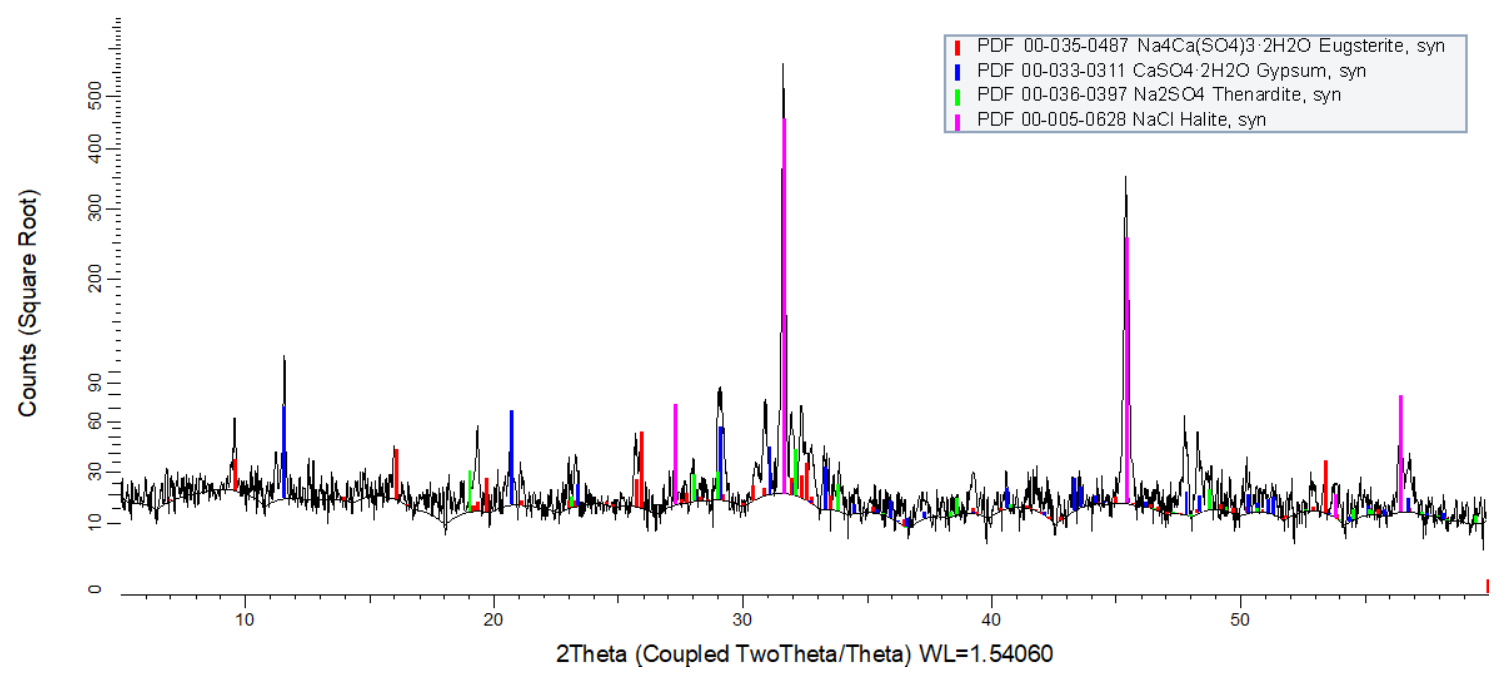

Figure S8: Representative diffractogram of the salt efflorescences made of chlorides, sulphates and borates in the emerged areas. The sample is composed of gypsum, eugsterite, halite and some thenardite.

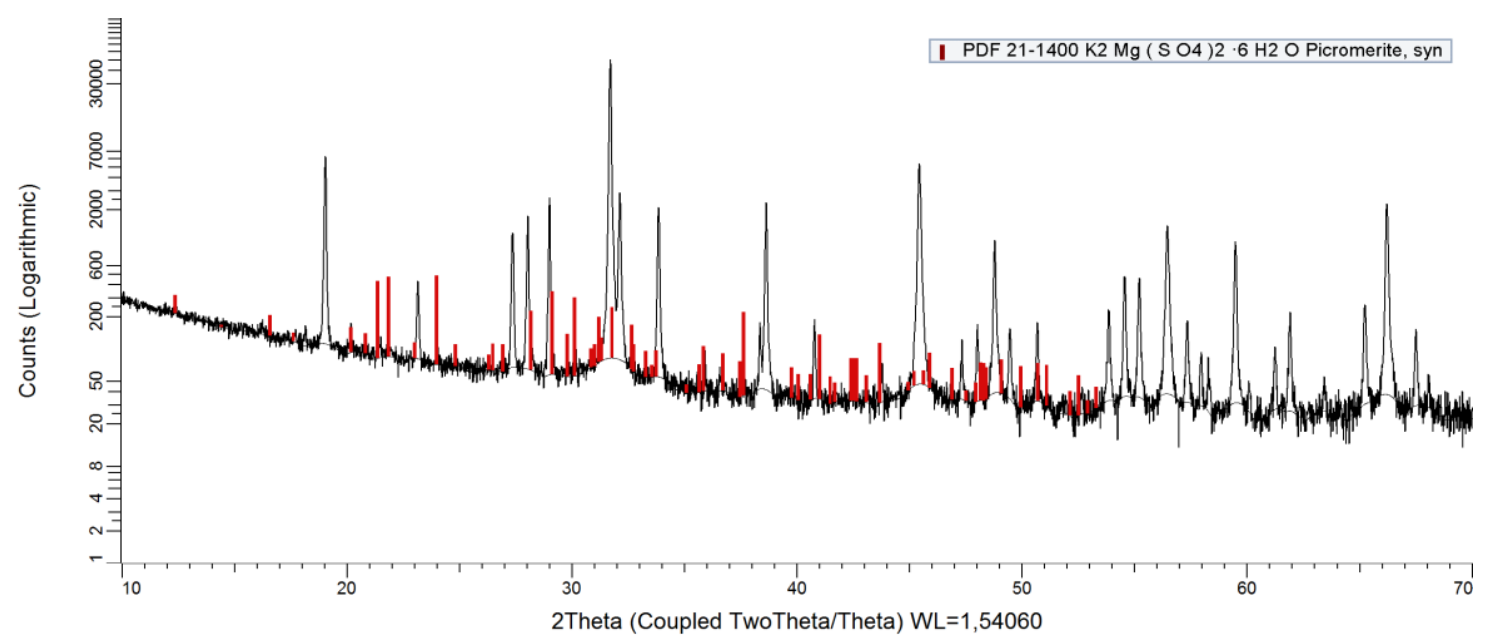

Figure S9: Diffractogram from the salt efflorescences showing the presence of Picromerite $\left(\mathrm{K}_{2} \mathrm{Mg}\left(\mathrm{SO}_{4}\right) 6 \mathrm{H}_{2} \mathrm{O}\right)$, a mineral not previously reported in this area

\section{CHEMISTRY OF WATERS}


Table S4: Identification, sampling date and position of the samples analyzed for chemical composition. The temperature, $\mathrm{pH}, \mathrm{ORP}, \mathrm{OD}$ and conductivity (corresponding to TDS) are duplicated from table S2 for easy cross referencing with the chemical data shown below.

\begin{tabular}{|c|c|c|c|c|c|c|c|c|c|}
\hline Code & pos & date & latitude & longitude & temp. & $\mathrm{pH}$ & ORP & OD & cond \\
\hline P11-1 & $\mathrm{S}$ & $28 / 10 / 11$ & $21^{\circ} 16^{\prime} 09^{\prime \prime}$ & $69^{\circ} 37^{\prime} 06^{\prime \prime}$ & 25.02 & 8.47 & -27.1 & 45.8 & 37540 \\
\hline P11-2 & $\mathrm{B}$ & $28 / 10 / 11$ & $21^{\circ} 16^{\prime} 05^{\prime \prime} 5$ & $69^{\circ} 37^{\prime} 06^{\prime \prime}$ & 43.13 & 7.30 & 26.7 & 162.0 & 104300 \\
\hline P11-3 & $\mathrm{T}$ & $28 / 10 / 11$ & $21^{\circ} 16^{\prime} 05^{\prime \prime} 5$ & $69^{\circ} 37^{\prime} 06^{\prime \prime}$ & 23.77 & 8.22 & 22.2 & 121.6 & 51410 \\
\hline P11-4 & $\mathrm{B}$ & $28 / 10 / 11$ & $21^{\circ} 16^{\prime} 06^{\prime \prime}$ & $69^{\circ} 37^{\prime} 04^{\prime \prime} 2$ & 47.42 & 7.25 & -184.9 & 37.4 & 148500 \\
\hline P11-5 & $\mathrm{T}$ & $28 / 10 / 11$ & $21^{\circ} 16^{\prime} 06^{\prime \prime}$ & $69^{\circ} 37^{\prime} 04^{\prime \prime} 2$ & 24.02 & 8.18 & -15.0 & 50.4 & 65960 \\
\hline P11-6 & B & $28 / 10 / 11$ & $21^{\circ} 16^{\prime} 07^{\prime \prime} 9$ & $69^{\circ} 37^{\prime} 02^{\prime \prime} 2$ & 41.10 & 7.22 & -193.9 & 58.8 & 149500 \\
\hline P11-7 & $\mathrm{T}$ & $28 / 10 / 11$ & $21^{\circ} 16^{\prime} 07^{\prime \prime} 9$ & $69^{\circ} 37^{\prime} 02^{\prime \prime} 2$ & 24.65 & 7.55 & -88.2 & 38.5 & 107100 \\
\hline P11-8 & $\mathrm{T}$ & $28 / 10 / 11$ & $21^{\circ} 16^{\prime} 06^{\prime \prime} 8$ & $69^{\circ} 37^{\prime} 02^{\prime \prime} 4$ & 25.36 & 7.82 & 15.6 & 73.4 & 145200 \\
\hline P11-9 & $\mathrm{B}$ & $28 / 10 / 11$ & $21^{\circ} 16^{\prime} 06^{\prime \prime} 3$ & $69^{\circ} 37^{\prime} 02^{\prime \prime} 8$ & 24.67 & 7.86 & 45.2 & 40.6 & 145000 \\
\hline P11-10 & $\mathrm{S}$ & $28 / 10 / 11$ & $21^{\circ} 16^{\prime} 06^{\prime \prime} 5$ & $69^{\circ} 37^{\prime} 01^{\prime \prime} 7$ & 23.61 & 8.12 & 172.3 & 56.0 & 134900 \\
\hline P12-1 & $\mathrm{S}$ & $22 / 03 / 12$ & $21^{\circ} 16^{\prime} 09^{\prime \prime}$ & $69^{\circ} 37^{\prime} 06^{\prime \prime}$ & 29.10 & 8.30 & -230.4 & 104.6 & 38280 \\
\hline P12-2 & $\mathrm{B}$ & $22 / 03 / 12$ & $21^{\circ} 16^{\prime} 05,5$ & $69^{\circ} 37^{\prime} 06^{\prime \prime}$ & 43.12 & 6.96 & -228.5 & 169.6 & 121900 \\
\hline P12-3 & $\mathrm{T}$ & $22 / 03 / 12$ & $21^{\circ} 16^{\prime} 05,5$ & $69^{\circ} 37^{\prime} 06^{\prime \prime}$ & 26.40 & 7.89 & -203.1 & 120.0 & 65430 \\
\hline P12-4 & $\mathrm{B}$ & $22 / 03 / 12$ & $21^{\circ} 16^{\prime} 06^{\prime \prime}$ & $69^{\circ} 37^{\prime} 04,2$ & 38.26 & 7.46 & -194.4 & 194.6 & 160000 \\
\hline P12-5 & $\mathrm{T}$ & $22 / 03 / 12$ & $21^{\circ} 16^{\prime} 06^{\prime \prime}$ & $69^{\circ} 37^{\prime} 04,2$ & 25.57 & 8.20 & -191.2 & 104.5 & 76170 \\
\hline P12-6 & $\mathrm{B}$ & $22 / 03 / 12$ & $21^{\circ} 16^{\prime} 07,9$ & $69^{\circ} 37^{\prime} 02^{\prime \prime 2}$ & 39.84 & 7.59 & -158.2 & 218.0 & 166500 \\
\hline P12-7 & $\mathrm{T}$ & $22 / 03 / 12$ & $21^{\circ} 16^{\prime} 07,9$ & $69^{\circ} 37^{\prime} 02,2$ & 26.72 & 8.13 & -170.0 & 99.5 & 101800 \\
\hline P12-8b & B & $22 / 03 / 12$ & $21^{\circ} 16^{\prime} 06,8$ & $69^{\circ} 37^{\prime} 02,4$ & 36.46 & 7.61 & -164.9 & 99.1 & 168200 \\
\hline P12-8 & $\mathrm{T}$ & $22 / 03 / 12$ & $21^{\circ} 16^{\prime} 06,8$ & $69^{\circ} 37^{\prime} 02,4$ & 28.05 & 7.90 & -165.4 & 91.1 & 155400 \\
\hline $\mathrm{P} 12-9 \mathrm{~b}$ & $\mathrm{~B}$ & $22 / 03 / 12$ & $21^{\circ} 16^{\prime} 06,3$ & $69^{\circ} 37^{\prime} 02,8$ & 35.84 & 7.55 & -153.4 & 86.0 & 172800 \\
\hline P12-9 & $\mathrm{T}$ & $22 / 03 / 12$ & $21^{\circ} 16^{\prime} 06,3$ & $69^{\circ} 37^{\prime} 02,8$ & 28.16 & 7.93 & -158.1 & 90.0 & 155500 \\
\hline P12-10 & $\mathrm{S}$ & $22 / 03 / 12$ & $21^{\circ} 16^{\prime} 06,5$ & $69^{\circ} 37^{\prime} 01,7$ & 30.89 & 7.97 & -175.7 & 62.9 & 178100 \\
\hline
\end{tabular}

Table S5: Major elements composition $\left(\mathrm{Cl}, \mathrm{SO}_{4}, \mathrm{HCO}_{3}, \mathrm{~S}, \mathrm{Na}, \mathrm{K}, \mathrm{Ca}, \mathrm{Mg}\right)$ of the brines analyzed

\begin{tabular}{lllllllll} 
Code & $\mathrm{Cl}$ & $\mathrm{SO}_{4}$ & $\mathrm{HCO}_{3}$ & $\mathrm{~S}$ & $\mathrm{Na}$ & $\mathrm{K}$ & $\mathrm{Ca}$ & $\mathrm{Mg}$ \\
\hline $\mathrm{P} 11-1$ & 10448 & 7410 & 113 & & 9406 & 244 & 882 & 177 \\
\hline $\mathrm{P} 11-2$ & 26508 & 20490 & 120 & & 26955 & 628 & 646 & 528 \\
\hline $\mathrm{P} 11-3$ & 15000 & 8921 & 137 & & 12866 & 346 & 763 & 238 \\
\hline $\mathrm{P} 11-4$ & 35874 & 21125 & 146 & & 32556 & 818 & 623 & 601 \\
\hline $\mathrm{P} 11-5$ & 19925 & 11541 & 161 & & 18544 & 479 & 792 & 327 \\
\hline $\mathrm{P} 11-6$ & 70603 & 26300 & 137 & & 53603 & 1437 & 563 & 916 \\
\hline P11-7 & 24181 & 11436 & 151 & & 19483 & 509 & 890 & 333 \\
\hline P11-8 & 64230 & 25780 & 191 & & 60347 & 1607 & 803 & 1000 \\
\hline P11-9 & 64276 & 25218 & 184 & & 48970 & 1319 & 644 & 834 \\
\hline P11-10 & 65409 & 26115 & 210 & & 49255 & 1306 & 651 & 836 \\
\hline P12-1 & 13597 & 4298 & 155 & 2361 & 9835 & 269 & 1027 & 192 \\
\hline P12-2 & 40472 & 21134 & 169 & 7054 & 31483 & 781 & 734 & 551 \\
\hline P12-3 & 20511 & 10035 & 169 & 3350 & 16327 & 462 & 939 & 276 \\
\hline P12-4 & 61159 & 30366 & 182 & 10136 & 51157 & 1307 & 610 & 840 \\
\hline P12-5 & 26328 & 12311 & 207 & 4109 & 21252 & 568 & 900 & 359 \\
\hline P12-6 & 73503 & 29530 & 207 & 9857 & 54211 & 1467 & 596 & 863 \\
\hline P12-7 & 39859 & 15071 & 256 & 5031 & 28961 & 802 & 949 & 459 \\
\hline
\end{tabular}




\begin{tabular}{lllllllll}
\hline P12-8b & 77987 & 29533 & 233 & 9858 & 56343 & 1581 & 561 & 904 \\
\hline P12-8 & 71144 & 13843 & 234 & 8215 & 49487 & 1441 & 705 & 809 \\
\hline P12-9b & 90956 & 20088 & 220 & 10976 & 59948 & 1645 & 535 & 935 \\
\hline P12-9 & 71270 & 16162 & 235 & 8748 & 50449 & 1416 & 651 & 808 \\
\hline P12-10 & 82380 & 20464 & 290 & 11277 & 62292 & 1727 & 549 & 975 \\
\hline
\end{tabular}

Table S6: Minor and trace elements composition (Mn, Sr, B, Li, Si) of the brines analyzed

\begin{tabular}{llllll} 
code & $\mathrm{Mn}$ & $\mathrm{Sr}$ & $\mathrm{B}$ & $\mathrm{Li}$ & $\mathrm{Si}$ \\
\hline $\mathrm{P} 12-1$ & & 8 & 10 & 3 & 179 \\
\hline $\mathrm{P} 12-2$ & 5 & 15 & 33 & 9 & 156 \\
\hline $\mathrm{P} 12-3$ & & 19 & 15 & 5 & 192 \\
\hline $\mathrm{P} 12-4$ & 1 & 16 & 54 & 15 & 139 \\
\hline $\mathrm{P} 12-5$ & & 11 & 26 & 6 & 181 \\
\hline $\mathrm{P} 12-6$ & 2 & 14 & 53 & 16 & 140 \\
\hline $\mathrm{P} 12-7$ & & 9 & 26 & 8 & 183 \\
\hline $\mathrm{p} 12-8 \mathrm{~b}$ & & 14 & 56 & 16 & 131 \\
\hline $\mathrm{P} 12-8$ & & 16 & 47 & 15 & 151 \\
\hline $\mathrm{P} 12-9 \mathrm{~b}$ & 13 & 62 & 17 & 132 \\
\hline $\mathrm{P} 12-9$ & & 16 & 49 & 15 & 146 \\
\hline $\mathrm{P} 12-10$ & 20 & 63 & 18 & 149 \\
\hline
\end{tabular}

Table S7: Isotopic composition $\left(\delta^{18} \mathrm{O}, \delta \mathrm{D}, \delta^{34} \mathrm{~S}, \delta^{18} \mathrm{O}\right)$ of the brines analyzed.

\begin{tabular}{lllll} 
code & $\delta 18 \mathrm{O}$ & $\delta \mathrm{D}$ & $\delta 34 \mathrm{~S}$ & $\delta 18 \mathrm{O}$ \\
\hline $\mathrm{P} 2-1$ & -1.1 & -33.5 & 4.0 & 12.0 \\
\hline $\mathrm{P} 2-2$ & 0.5 & -24.0 & 3.5 & 11.4 \\
\hline $\mathrm{P} 2-3$ & -0.1 & -29.7 & 3.7 & 11.4 \\
\hline $\mathrm{P} 2-4$ & 3.9 & -10.3 & 3.7 & 11.4 \\
\hline $\mathrm{P} 2-5$ & 6.3 & 7.5 & 3.5 & 10.9 \\
\hline $\mathrm{P} 2-6$ & 6.7 & 9.1 & 3.6 & 10.7 \\
\hline $\mathrm{P} 2-7$ & 2.7 & -15.4 & 3.7 & 11.2 \\
\hline $\mathrm{P} 2-8$ & 9.4 & 18.7 & 3.3 & 10.4 \\
\hline $\mathrm{P} 2-8 \mathrm{~b}$ & 9.5 & 21.1 & 3.6 & 10.4 \\
\hline $\mathrm{P} 2-9$ & 9.6 & 20.3 & 3.5 & 10.6 \\
\hline $\mathrm{P} 2-9 \mathrm{~b}$ & 5.3 & 1.8 & 3.5 & 10.6 \\
\hline $\mathrm{P} 2-10$ & 5.1 & 0.1 & 3.5 & 10.7 \\
\hline
\end{tabular}

Table S8: Saturation indices calculated (PHREEQC, Pitzer database ${ }^{5}$ ) for different phases (halite, gypsum, anhydrite, calcite, aragonite, eugsterite, glauberite, thenartdite, sylvite) in the brines analyzed. The total ionic strength $(\mu)$ is also shown.

\begin{tabular}{|l|l|l|l|l|l|l|l|l|l|l|}
\hline code & $\mu$ & halite & Gyps. & Anhyd. & calcite & Arag. & Eeugs. & Glaub. & Thenar. & Ssylvite \\
\hline P11-1 & 0.5860 & -2.8394 & 0.1236 & -0.2141 & 1.3717 & 1.0897 & -5.0849 & -2.2552 & -2.8396 & -4.0012 \\
\hline P11-2 & 1.5911 & -2.0218 & 0.0256 & -0.0814 & -1.0472 & -1.3743 & -3.1244 & -1.1142 & -1.7041 & -3.3975 \\
\hline P11-3 & 0.7693 & -2.5543 & 0.0513 & -0.2974 & 0.7879 & 0.5105 & -4.6234 & -2.0664 & -2.5791 & -3.6966 \\
\hline P11-4 & 1.9211 & -1.7867 & 0.0002 & -0.0505 & -1.0796 & -1.4124 & -2.8918 & -0.9462 & -1.5464 & -3.1703 \\
\hline P11-5 & 1.0523 & -2.2787 & 0.0556 & -0.2836 & 0.7084 & 0.4301 & -3.9588 & -1.7236 & -2.2477 & -3.4503 \\
\hline P11-6 & 3.3446 & -1.1361 & 0.0269 & -0.0496 & -0.9641 & -1.2879 & -1.8796 & -0.4628 & -1.0955 & -2.5060 \\
\hline P11-7 & 1.1466 & -2.1697 & 0.0961 & -0.2326 & -0.4591 & -0.7398 & -3.8963 & -1.6642 & -2.2335 & -3.3470 \\
\hline
\end{tabular}




\begin{tabular}{|l|l|l|l|l|l|l|l|l|l|l|}
\hline P11-8 & 3.4257 & -1.1066 & 0.1096 & -0.1486 & 0.2481 & -0.0352 & -1.6680 & -0.4755 & -1.1224 & -2.3570 \\
\hline P11-9 & 3.0453 & -1.2343 & 0.0792 & -0.1994 & 0.2238 & -0.0569 & -2.0617 & -0.7054 & -1.3077 & -2.4649 \\
\hline P11-10 & 3.1022 & -1.2202 & 0.0997 & -0.1909 & 0.7456 & 0.4689 & -2.0126 & -0.6781 & -1.2988 & -2.4503 \\
\hline P12-1 & 0.5835 & -2.7017 & -0.0060 & -0.2929 & 1.2116 & 0.9159 & -5.6265 & -2.5532 & -3.0238 & -3.8769 \\
\hline P12-2 & 1.9758 & -1.7379 & 0.0987 & 0.0029 & -1.5621 & -1.8892 & -2.8447 & -0.9266 & -1.6009 & -3.1039 \\
\hline P12-3 & 0.9766 & -2.3213 & 0.1159 & -0.1955 & 0.2597 & -0.0272 & -4.1984 & -1.7946 & -2.3853 & -3.4726 \\
\hline P12-4 & 3.1879 & -1.2503 & 0.0717 & -0.0457 & -0.5599 & -0.8785 & -1.8249 & -0.4554 & -1.1084 & -2.6097 \\
\hline P12-5 & 1.2497 & -2.0953 & 0.1035 & -0.2116 & 0.8584 & 0.5744 & -3.7260 & -1.5653 & -2.1472 & -3.2737 \\
\hline P12-6 & 3.5136 & -1.1033 & 0.0973 & 0.0101 & -0.1957 & -0.5173 & -1.6942 & -0.3465 & -1.0460 & -2.4657 \\
\hline P12-7 & 1.7532 & -1.7594 & 0.1393 & -0.1486 & 0.8215 & 0.5335 & -3.1598 & -1.2411 & -1.8760 & -2.9518 \\
\hline p12-8b & 3.6775 & -1.0382 & 0.0864 & -0.0330 & -0.1583 & -0.4732 & -1.6237 & -0.3491 & -1.0262 & -2.3684 \\
\hline P12-8 & 2.8857 & -1.1651 & -0.0564 & -0.2898 & 0.4782 & 0.1858 & -2.6152 & -1.0163 & -1.4987 & -2.3950 \\
\hline P12-9b & 3.7903 & -0.8955 & -0.0033 & -0.1165 & -0.1477 & -0.4612 & -1.8657 & -0.5084 & -1.1061 & -2.2467 \\
\hline P12-9 & 2.9759 & -1.1543 & -0.0401 & -0.2709 & 0.4948 & 0.2020 & -2.4429 & -0.9197 & -1.4201 & -2.4026 \\
\hline P12-10 & 3.6963 & -0.9339 & -0.0425 & -0.2179 & 0.6018 & 0.3008 & -1.8634 & -0.5825 & -1.1140 & -2.2342 \\
\hline
\end{tabular}

\section{KINETIC COEFFICIENTS FITTED TO THE EXPERIMENTAL PRECIPITATION SEQUENCE}

Table S9: Values of the precipitation $\left(\mathrm{k}_{\mathrm{pre}}\right)$ and dissolution $\left(\mathrm{k}_{\mathrm{dis}}\right)$ kinetic parameters used in the calculation of the precipitation sequence (figure 15 in main text). All of them are accurate to the order of magnitude.

\begin{tabular}{lll} 
phase & $\mathrm{k}_{\text {pre }}$ & $\mathrm{k}_{\text {dis }}$ \\
\hline gypsum & $2 \cdot 10^{-7}$ & $2 \cdot 10^{-6}$ \\
\hline eugsterite & $2 \cdot 10^{-4}$ & $2 \cdot 10^{-3}$ \\
\hline glauberite & $2 \cdot 10^{-8}$ & $4 \cdot 10^{-8}$ \\
\hline halite & $2 \cdot 10^{-5}$ & $2 \cdot 10^{-4}$ \\
\hline thenardite & $2 \cdot 10^{-6}$ & $2 \cdot 10^{-5}$ \\
\hline
\end{tabular}

\section{REFERENCES}

1. Hartley, A. J. C. G., Late Pliocene age for the Atacama Desert: Implications for the desertification of western South America. Geology 2002, 30, 43-46. 
2. Jayne, R. S.; Pollyea, R. M.; Dodd, J. P.; Olson, E. J.; Swanson, S. K., Spatial and temporal constraints on regional-scale groundwater flow in the Pampa del Tamarugal Basin, Atacama Desert, Chile. Hydrogeol. J. 2016, 24, 1921-1937.

3. Houston, J., The great Atacama flood of 2001 and its implications for Andean hydrology. Hydrol. Processes 2006, 20, 591-610.

4. Tukey, J. W., Exploratory data analysis. Reading, Mass.: 1977, Vol. 2.

5. Parkhurst, D. L.; Appelo, C. A. J., Description of Input and Examples for PHREEQC Version 3-A Computer Program for Speciation, Batch-Reaction, OneDimensional Transport, and Inverse Geochemical Calculations. 2013, Vol. Book 6, chap. A43, p 497 p. 